Ind. Eng. Chem. Res.

\title{
Separation of linalool from limonene via extractive distillation with 1- butyl-3-methylimidazolium acetate as entrainer
}

Fernanda Ganem ${ }^{1}$, Silvana Mattedi ${ }^{1}$, Eva Rodil ${ }^{2}$ and Ana Soto ${ }^{2}$

${ }^{1}$ Chemical Engineering Graduate Program, Polytechnic School, Federal University of

Bahia, Rua Aristides Novis 2, Federação, 40210-630 Salvador-BA, Brazil

${ }^{2}$ CRETUS Institute, Chemical Engineering Department - ETSE, Universidade de Santiago de Compostela, E-15782 Santiago de Compostela, Spain

\section{Supplementary Material}

Index:

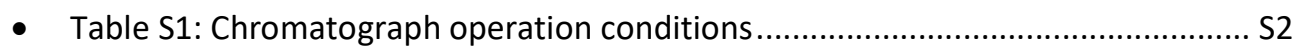

- Table S2: Parameters used in the definition of the ionic liquid in Aspen Plus.......... S2

- Table S3: Vapor-liquid equilibrium data for the binary system ................................S2

- Table S4: Vapor-liquid equilibrium data for ternary system..................................... S3

- Table S5: Simulation results: Separation of limonene and linalool by extractive distillation $(5 \mathrm{kPa})$ with $\left[\mathrm{C}_{2} \mathrm{mim}\right][\mathrm{OAc}]$ using a flash unit $(0.1 \mathrm{kPa}, 423 \mathrm{~K})$ to

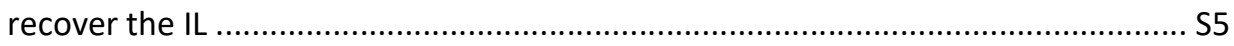

- Figure S1: ${ }^{1} \mathrm{H}$ and ${ }^{13} \mathrm{C}$ spectra of the purified ionic liquid ......................................... S6

- Figure S2. Linalool and limonene recovery and purity, heat duty and cooling requirements, as a function of the number of stages and reflux ratio in the extractive distillation column ( $\mathrm{S} / \mathrm{F}=0.025)$, according to the schematic flowsheet shown in Figure 1 (main text).

- Figure S3. Linalool and limonene recovery and purity, heat duty and cooling requirements, as a function of the number of stages and reflux ratio in the extractive distillation column ( $\mathrm{S} / \mathrm{F}=0.05)$, according to the schematic flowsheet shown in Figure 1 (main text).

- Figure S4. Linalool and limonene recovery and purity, heat duty and cooling requirements, as a function of the number of stages and reflux ratio in the extractive distillation column ( $\mathrm{S} / \mathrm{F}=0.025)$, according to the schematic flowsheet shown in Figure 2 (main text)

- Figure S5. Linalool and limonene recovery and purity, heat duty and cooling requirements, as a function of the number of stages and reflux ratio in the extractive distillation column ( $S / F=0.05)$, according to the schematic flowsheet shown in Figure 2 (main text)

- References. 
Table S1. Chromatograph operating conditions.

\begin{tabular}{|c|c|}
\hline Column* & HP-5 $(30 \mathrm{~m} \times 0.32 \mathrm{~mm} \times 0.25 \mu \mathrm{m})$ \\
\hline Detector type & FID \\
\hline Carrier gas & Helium (split ratio 50:1) \\
\hline Injector temperature & $523.15 \mathrm{~K}$, injection volume $1 \mu \mathrm{L}$ \\
\hline Detector temperature & $523.15 \mathrm{~K}$ \\
\hline Flow rate & $1 \mathrm{ml} \mathrm{min}^{-1}$ \\
\hline \multirow[t]{3}{*}{ Column oven } & $363.15 \mathrm{~K}(13.5 \mathrm{~min})$ \\
\hline & Ramp, $60 \mathrm{~K} \cdot \mathrm{min}^{-1}$ up to $493.15 \mathrm{~K}$ \\
\hline & $493.15 \mathrm{~K}(0.5 \mathrm{~min})$ \\
\hline
\end{tabular}

Table S2. Parameters used in the definition of the ionic liquid $\left[\mathrm{C}_{4} \mathrm{mim}\right][\mathrm{OAc}]$, as userdefined compound, in the simulation software Aspen Plus.

\begin{tabular}{|c|c|}
\hline Parameter & {$\left[\mathrm{C}_{4} \mathrm{mim}\right][\mathrm{OAc}]$} \\
\hline Molar mass (g/mol) & 198.64 \\
\hline \multicolumn{2}{|l|}{ Critical properties ${ }^{[\mathrm{S} 1]}$} \\
\hline Critical temperature, $T_{c}(\mathrm{~K})$ & 847.3 \\
\hline Critical pressure, $P_{c}$ (bar) & 24.5 \\
\hline Critical molar volume, $V_{c}\left(\mathrm{~cm}^{3} / \mathrm{mol}\right)$ & 658.2 \\
\hline Critical compressibility factor, $Z_{c}$ & 0.2289 \\
\hline Acentric factor, $\omega$ & 0.6681 \\
\hline \multicolumn{2}{|l|}{ Vapor pressure $\left(P^{v}\right)$ correlation parameters ${ }^{a *}$} \\
\hline $\mathrm{C}_{1}$ & 11.55 \\
\hline $\mathrm{C}_{2}$ & -6718.81 \\
\hline $\mathrm{C}_{3}$ & -43 \\
\hline \multicolumn{2}{|l|}{ Heat capacity $\left(C_{p}\right)$ correlation parameters ${ }^{b}[\mathrm{~S} 2]$} \\
\hline $\mathrm{C}_{1}$ & 310.23 \\
\hline $\mathrm{C}_{2}$ & -0.1084 \\
\hline $\mathrm{C}_{3}$ & 0.0010 \\
\hline $\mathrm{C}_{4}, \mathrm{C}_{5}, \mathrm{C}_{6}$ & 0 \\
\hline $\mathrm{C}_{7}$ (lower end of valid temperature range, $\mathrm{K}$ ) & 273 \\
\hline $\mathrm{C}_{8}$ (upper end of valid temperature range, $\mathrm{K}$ ) & 600 \\
\hline
\end{tabular}

*Vapor pressure parameters calculated by Antoine-Rudkin correlation [S3]

Table S3. Experimental vapor-liquid equilibrium data for the binary system linalool (1) $+\left[\mathrm{C}_{4} \mathrm{mim}\right][\mathrm{OAc}](2)$ at $5 \mathrm{kPa}$

\begin{tabular}{cccccc}
\hline$T / K$ & $x_{I} /$ (f.mol.) & $T / K$ & $x_{I} /$ (f.mol.) & $T / K$ & $x_{I} /$ (f.mol.) \\
\hline 382.85 & 1.0000 & 391.96 & 0.7505 & 406.85 & 0.5102 \\
383.45 & 0.9749 & 393.99 & 0.7298 & 409.05 & 0.4914 \\
384.44 & 0.9148 & 395.95 & 0.7052 & 412.15 & 0.4354 \\
385.50 & 0.8865 & 398.44 & 0.6597 & 414.25 & 0.4070 \\
386.61 & 0.8648 & 401.12 & 0.6044 & 419.06 & 0.3589 \\
388.64 & 0.8158 & 402.41 & 0.5854 & 421.12 & 0.3349 \\
389.59 & 0.7981 & 404.44 & 0.5508 & 422.69 & 0.3159
\end{tabular}

Uncertainties: $u(T)=0.1 \mathrm{~K} . u(P)=0.005 \mathrm{kPa} . u\left(x_{l}\right)=0.003$. 
Table S4. Equilibrium temperatures $(T)$ and liquid and vapor mole fractions $\left(x_{1}, y_{1}\right)$ for limonene (1) + linalool (2) $+\left[\mathrm{C}_{4} \mathrm{mim}\right][\mathrm{OAc}](3)$ at $5 \mathrm{kPa}$.

\begin{tabular}{|c|c|c|c|c|}
\hline$T / \mathrm{K}$ & $x_{1} /($ f.mol. $)$ & $x_{2} /($ f.mol. $)$ & $y_{1} /($ f.mol. $)$ & $y_{2} /($ f.mol. $)$ \\
\hline 362.43 & 0.0820 & 0.3157 & 0.9947 & 0.0053 \\
\hline 362.64 & 0.3111 & 0.4022 & 0.9625 & 0.0375 \\
\hline 364.05 & 0.2864 & 0.3902 & 0.9583 & 0.0417 \\
\hline 364.21 & 0.2848 & 0.4039 & 0.9451 & 0.0549 \\
\hline 364.45 & 0.5614 & 0.4054 & 0.8640 & 0.1360 \\
\hline 364.85 & 0.3218 & 0.4717 & 0.8700 & 0.1300 \\
\hline 365.39 & 0.2875 & 0.4559 & 0.9358 & 0.0642 \\
\hline 365.40 & 0.4941 & 0.4314 & 0.8443 & 0.1557 \\
\hline 365.61 & 0.4590 & 0.4595 & 0.8423 & 0.1577 \\
\hline 365.86 & 0.4254 & 0.4650 & 0.8403 & 0.1597 \\
\hline 365.92 & 0.2316 & 0.4153 & 0.9613 & 0.0387 \\
\hline 366.26 & 0.2448 & 0.4438 & 0.9852 & 0.0148 \\
\hline 366.61 & 0.3782 & 0.4925 & 0.8179 & 0.1821 \\
\hline 366.93 & 0.3512 & 0.5109 & 0.8082 & 0.1918 \\
\hline 367.41 & 0.3167 & 0.5217 & 0.8099 & 0.1901 \\
\hline 368.08 & 0.1837 & 0.4137 & 0.9558 & 0.0442 \\
\hline 368.26 & 0.2774 & 0.5307 & 0.7955 & 0.2045 \\
\hline 368.75 & 0.1529 & 0.4205 & 0.9582 & 0.0418 \\
\hline 368.86 & 0.2521 & 0.5276 & 0.7929 & 0.2071 \\
\hline 369.52 & 0.1765 & 0.4558 & 0.9004 & 0.0996 \\
\hline 369.87 & 0.2213 & 0.5373 & 0.7779 & 0.2221 \\
\hline 370.24 & 0.1780 & 0.4799 & 0.8940 & 0.1060 \\
\hline 371.28 & 0.1979 & 0.5502 & 0.7593 & 0.2407 \\
\hline 372.64 & 0.1691 & 0.5401 & 0.7571 & 0.2429 \\
\hline 372.70 & 0.1802 & 0.6089 & 0.6662 & 0.3338 \\
\hline 373.17 & 0.1480 & 0.6233 & 0.6768 & 0.3232 \\
\hline 373.21 & 0.1596 & 0.6296 & 0.6751 & 0.3249 \\
\hline 374.19 & 0.1403 & 0.6331 & 0.6483 & 0.3517 \\
\hline 374.52 & 0.1370 & 0.5508 & 0.7318 & 0.2682 \\
\hline 374.63 & 0.1349 & 0.5560 & 0.7043 & 0.2957 \\
\hline 374.73 & 0.1315 & 0.5536 & 0.7189 & 0.2811 \\
\hline 374.73 & 0.1353 & 0.6405 & 0.6211 & 0.3789 \\
\hline 374.91 & 0.1331 & 0.5168 & 0.7853 & 0.2147 \\
\hline 375.33 & 0.1249 & 0.5471 & 0.6985 & 0.3015 \\
\hline 375.80 & 0.1177 & 0.6855 & 0.5615 & 0.4385 \\
\hline 375.95 & 0.1186 & 0.5675 & 0.6535 & 0.3465 \\
\hline 376.79 & 0.1019 & 0.6894 & 0.5199 & 0.4801 \\
\hline 377.69 & 0.0852 & 0.6421 & 0.4677 & 0.5323 \\
\hline 377.86 & 0.0973 & 0.5978 & 0.6354 & 0.3646 \\
\hline 378.39 & 0.0957 & 0.5664 & 0.6421 & 0.3579 \\
\hline 378.65 & 0.0796 & 0.7249 & 0.4162 & 0.5838 \\
\hline 378.96 & 0.0730 & 0.8021 & 0.3171 & 0.6829 \\
\hline 379.52 & 0.0681 & 0.7275 & 0.3578 & 0.6422 \\
\hline
\end{tabular}




\begin{tabular}{ccccc}
379.62 & 0.0832 & 0.5926 & 0.6075 & 0.3925 \\
379.89 & 0.0778 & 0.5919 & 0.5635 & 0.4365 \\
380.57 & 0.0741 & 0.6110 & 0.5335 & 0.4665 \\
380.78 & 0.0436 & 0.6609 & 0.3224 & 0.6776 \\
381.27 & 0.0400 & 0.8137 & 0.2041 & 0.7959 \\
381.58 & 0.0530 & 0.4238 & 0.7701 & 0.2299 \\
381.61 & 0.0349 & 0.8216 & 0.1799 & 0.8201 \\
381.82 & 0.0321 & 0.8502 & 0.1639 & 0.8361 \\
381.90 & 0.0671 & 0.6109 & 0.4478 & 0.5522 \\
382.36 & 0.0355 & 0.7649 & 0.2314 & 0.7686 \\
382.39 & 0.0135 & 0.8730 & 0.0614 & 0.9386 \\
382.76 & 0.0108 & 0.8296 & 0.0511 & 0.9489 \\
382.94 & 0.0094 & 0.8716 & 0.0440 & 0.9560 \\
384.61 & 0.0490 & 0.5973 & 0.4202 & 0.5798 \\
384.87 & 0.0535 & 0.4275 & 0.7554 & 0.2446 \\
386.27 & 0.0567 & 0.3993 & 0.8298 & 0.1702 \\
388.21 & 0.0522 & 0.5045 & 0.6731 & 0.3269 \\
388.56 & 0.0426 & 0.5898 & 0.4730 & 0.5270 \\
389.45 & 0.0439 & 0.5259 & 0.6031 & 0.3969 \\
391.25 & 0.0347 & 0.3013 & 0.7884 & 0.2116 \\
392.11 & 0.0316 & 0.3925 & 0.7848 & 0.2152 \\
396.66 & 0.0240 & 0.3709 & 0.7216 & 0.2784 \\
397.23 & 0.0221 & 0.4788 & 0.4784 & 0.5216 \\
399.10 & 0.0207 & 0.4260 & 0.5599 & 0.4401 \\
403.58 & 0.0161 & 0.3981 & 0.5448 & 0.4552 \\
405.10 & 0.0147 & 0.4218 & 0.4178 & 0.5822 \\
407.27 & 0.0107 & 0.4246 & 0.4145 & 0.5855 \\
407.43 & 0.0095 & 0.4664 & 0.5241 & 0.3444 \\
408.87 & 0.0103 & 0.3826 & 0.6071 & 0.3163 \\
410.13 & 0.0070 & 0.3911 & 0.6019 & 0.2375 \\
\hline$u(T)=0.1 \mathrm{~K} . u(P)=0.005 \mathrm{kPa} . u\left(x_{1}\right)=0.003 . u\left(x_{2}\right)=0.002 . u\left(y_{1}\right)=0.004 . u\left(y_{2}\right)=0.004$.
\end{tabular}


Table S5: Simulation results: Separation of limonene and linalool by extractive distillation $(5 \mathrm{kPa})$ with $\left[\mathrm{C}_{2} \mathrm{mim}\right][\mathrm{OAc}]$ using a flash unit $(0.1 \mathrm{kPa}, 423 \mathrm{~K})$ to recover the IL

\begin{tabular}{|c|c|c|c|c|}
\hline \multirow[b]{2}{*}{ Variable } & \multicolumn{4}{|c|}{ Solvent flowrate $(\mathrm{S}), \mathrm{kg} / \mathrm{h}$} \\
\hline & $0^{[16]}$ & 25 & 50 & 100 \\
\hline & \multicolumn{4}{|c|}{$90 \%$ Linalool purity } \\
\hline Reflux ratio $(\mathrm{R})$ & 1 & 0.5 & 0.5 & 0.5 \\
\hline Number of Stage $(\mathrm{N})$ & 21 & 15 & 10 & 7 \\
\hline Feed stage & 11 & 8 & 5 & 4 \\
\hline Linalool recovery $(\%)$ & 90.0 & 89.8 & 89.7 & 89.4 \\
\hline Limonene purity $(\%)$ & 98.9 & 98.9 & 98.9 & 98.8 \\
\hline Limonene recovery $(\%)$ & 98.9 & 98.9 & 98.9 & 98.9 \\
\hline Required heat duty (Q $\left.\mathrm{Q}_{\text {Heater }}\right), \mathrm{kW}$ & 200.0 & 172.7 & 174.5 & 178.0 \\
\hline \multirow[t]{2}{*}{ Required cooling duty $\left(\mathrm{Q}_{\text {cooling }}\right), \mathrm{kW}$} & 163.8 & 124.6 & 126.4 & 130.0 \\
\hline & \multicolumn{4}{|c|}{$95 \%$ Linalool purity } \\
\hline Reflux ratio $(\mathrm{R})$ & 1.5 & 1 & 0.5 & 0.5 \\
\hline Number of Stage $(\mathrm{N})$ & 20 & 15 & 14 & 9 \\
\hline Feed stage & 10 & 8 & 7 & 5 \\
\hline Linalool recovery $(\%)$ & 95.1 & 94.8 & 94.7 & 94.3 \\
\hline Limonene purity $(\%)$ & 99.5 & 99.4 & 99.4 & 99.4 \\
\hline Limonene recovery $(\%)$ & 99.5 & 99.4 & 99.4 & 99.4 \\
\hline Required heat duty (Q $\left.\mathrm{Q}_{\text {Heater }}\right), \mathrm{kW}$ & 241.0 & 213.6 & 174.5 & 178.0 \\
\hline \multirow{2}{*}{ Required cooling duty $\left(\mathrm{Q}_{\text {cooling }}\right), \mathrm{kW}$} & 204.6 & 165.4 & 126.3 & 130.0 \\
\hline & \multicolumn{4}{|c|}{$99.98 \%$ Linalool purity } \\
\hline Reflux ratio $(\mathrm{R})$ & 3 & 2.5 & 1 & 1 \\
\hline Number of Stage $(\mathrm{N})$ & 45 & 30 & 30 & 20 \\
\hline Feed stage & 23 & 15 & 15 & 10 \\
\hline Linalool recovery $(\%)$ & 100 & 99.8 & 99.6 & 99.2 \\
\hline Limonene purity $(\%)$ & 100 & 100.0 & 100.0 & 99.9 \\
\hline Limonene recovery $(\%)$ & 100 & 100.0 & 100.0 & 100.0 \\
\hline Required heat duty ( $\left.\mathrm{Q}_{\text {Heater }}\right), \mathrm{kW}$ & 363.7 & 336.1 & 215.3 & 178.0 \\
\hline Required cooling duty $\left(\mathrm{Q}_{\text {cooling }}\right), \mathrm{kW}$ & 327.0 & 288.0 & 167.1 & 129.9 \\
\hline
\end{tabular}




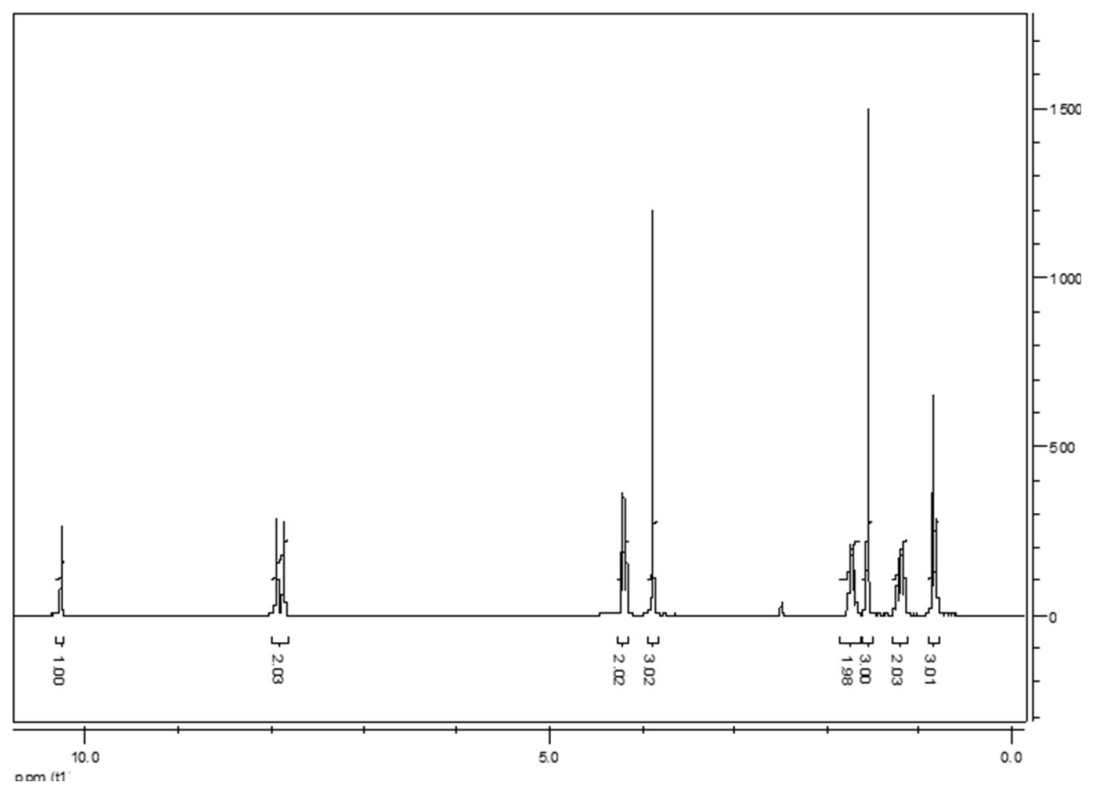

${ }^{1} \mathrm{H}$ NMR spectrum of [ $\left.\mathrm{C}_{4} \mathrm{mim}\right][\mathrm{OAc}] ; \delta_{H}\left(\mathrm{DMSO}-d_{6}, 300 \mathrm{MHz}\right): 0.86(\mathrm{t}, J=7.4 \mathrm{~Hz}, 3 \mathrm{H}$, $\mathrm{N}\left(\mathrm{CH}_{2}\right)_{3} \mathrm{C}_{3}$ ), 1.21 (sex, $J=7.4 \mathrm{~Hz}, 2 \mathrm{H}, \mathrm{N}\left(\mathrm{CH}_{2}\right)_{2} \mathrm{C}_{2}$ ), 1.57 (s, $3 \mathrm{H}, \mathrm{CH}_{3} \mathrm{COO}$ ), 1.74 (quin, $J=7.4 \mathrm{~Hz}$, $2 \mathrm{H}, \mathrm{NCH}_{2} \mathrm{CH}_{2}$ ), $3.88\left(\mathrm{~s}, 3 \mathrm{H}, \mathrm{NCH}_{3}\right), 4.20\left(\mathrm{q}, J=7.1 \mathrm{~Hz}, 2 \mathrm{H}, \mathrm{NCH}_{2}\right.$ ), 7.82-8.00 (unresolved, $2 \mathrm{H}$, $\mathrm{C}(5) \mathrm{H}$ and $\mathrm{C}(4) \mathrm{H}), 10.27(\mathrm{~s}, 1 \mathrm{H}, \mathrm{C}(2) \mathrm{H})$.

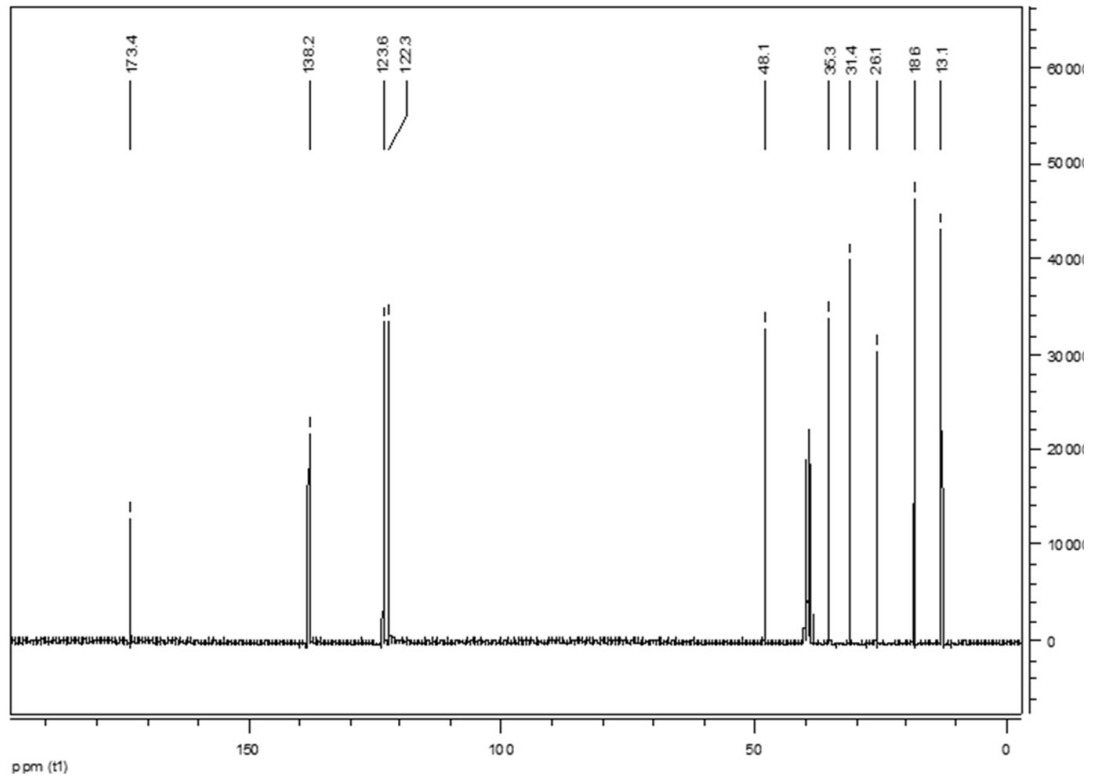

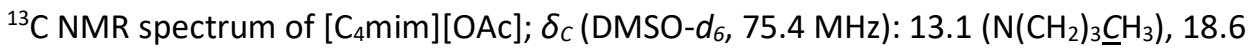
$\left.\left(\mathrm{N}\left(\mathrm{CH}_{2}\right)_{2} \underline{\mathrm{C}} \mathrm{H}_{2}\right), 26.1\left(\underline{\mathrm{C}} \mathrm{H}_{3} \mathrm{COO}\right), 31.4 \mathrm{NCH}_{2} \underline{\mathrm{C}} \mathrm{H}_{2}\right), 35.3\left(\mathrm{NCH}_{3}\right), 48.1\left(\mathrm{NCH}_{2}\right), 122.3(\mathrm{C}(4) \mathrm{H}), 123.6$ $(\mathrm{C}(5) \mathrm{H}), 138.2(\mathrm{C}(2) \mathrm{H}), 173.4\left(\mathrm{CH}_{3} \underline{\mathrm{COO}}\right)$.

Figure S1: ${ }^{1} \mathrm{H}$ and ${ }^{13} \mathrm{C}$ spectra of the purified ionic liquid 

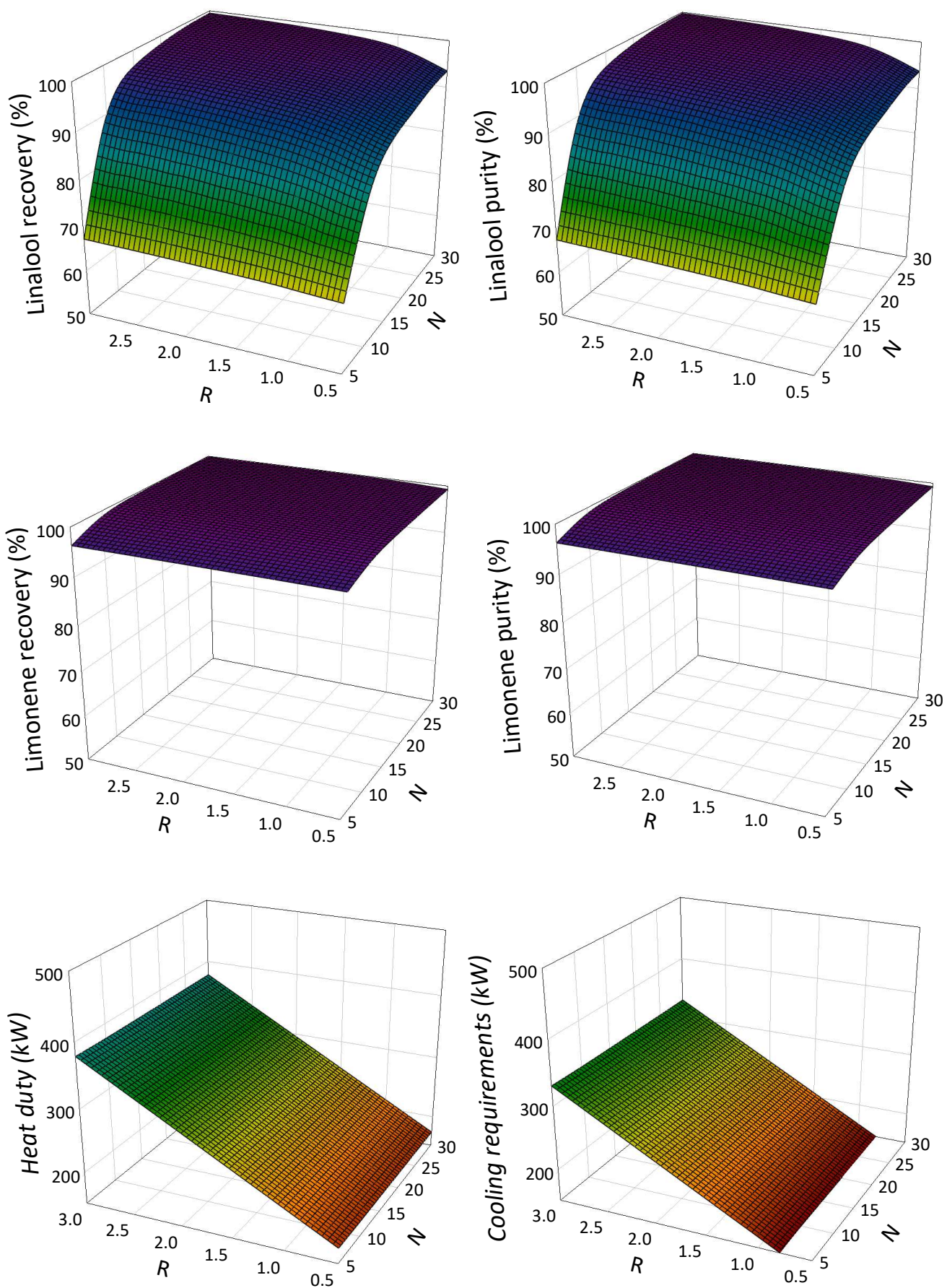

Figure S2. Linalool and limonene recovery and purity, heat duty and cooling requirements, as a function of the number of stages and reflux ratio in the extractive distillation column $(S / F=0.025)$, according to the schematic flowsheet shown in Figure 1 (main text). 

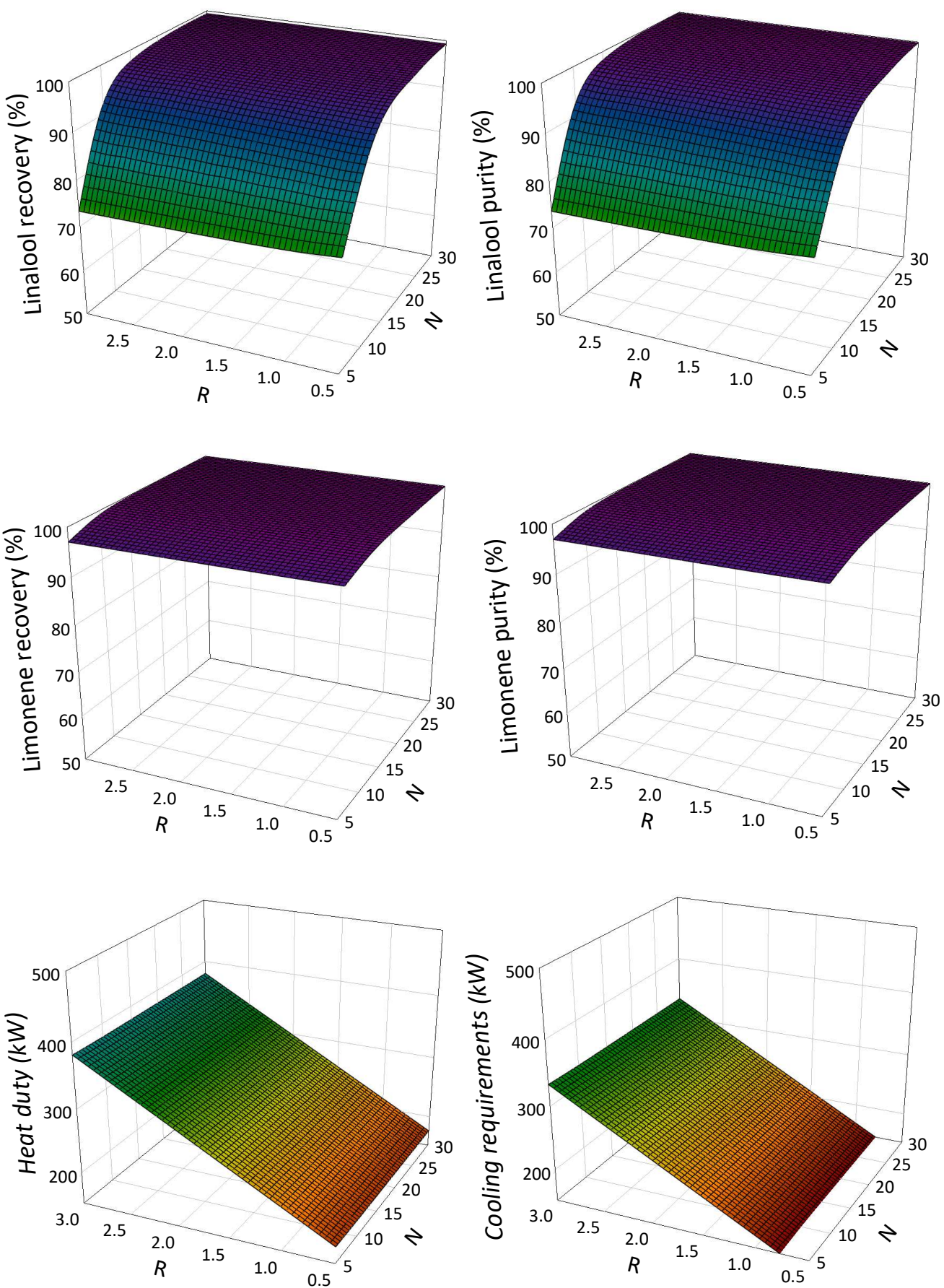

Figure S3. Linalool and limonene recovery and purity, heat duty and cooling requirements, as a function of the number of stages and reflux ratio in the extractive distillation column $(S / F=0.05)$, according to the schematic flowsheet shown in Figure 1 (main text). 

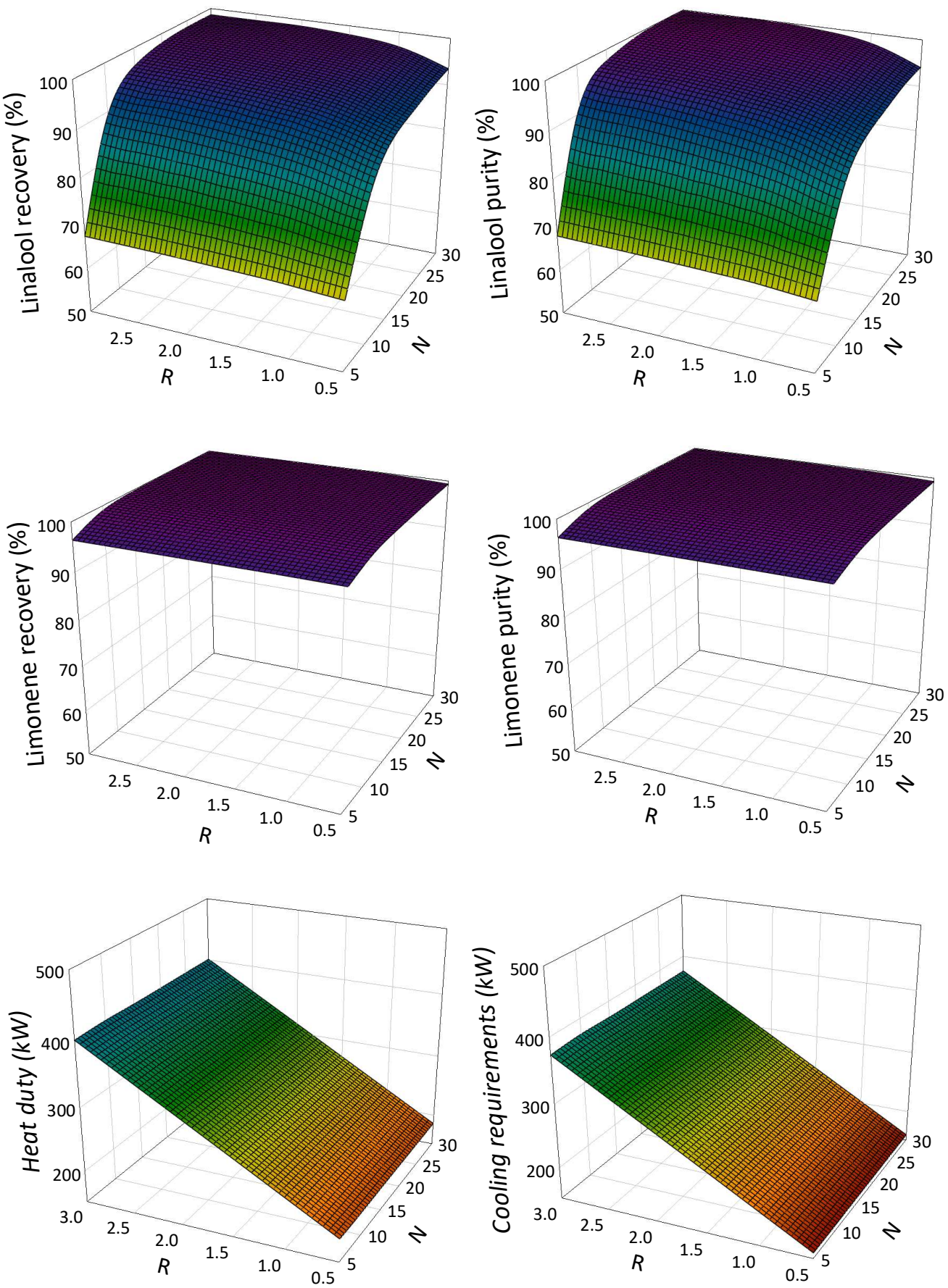

Figure S4. Linalool and limonene recovery and purity, heat duty and cooling requirements, as a function of the number of stages and reflux ratio in the extractive distillation column $(S / F=0.025)$, according to the schematic flowsheet shown in Figure 2 (main text). 

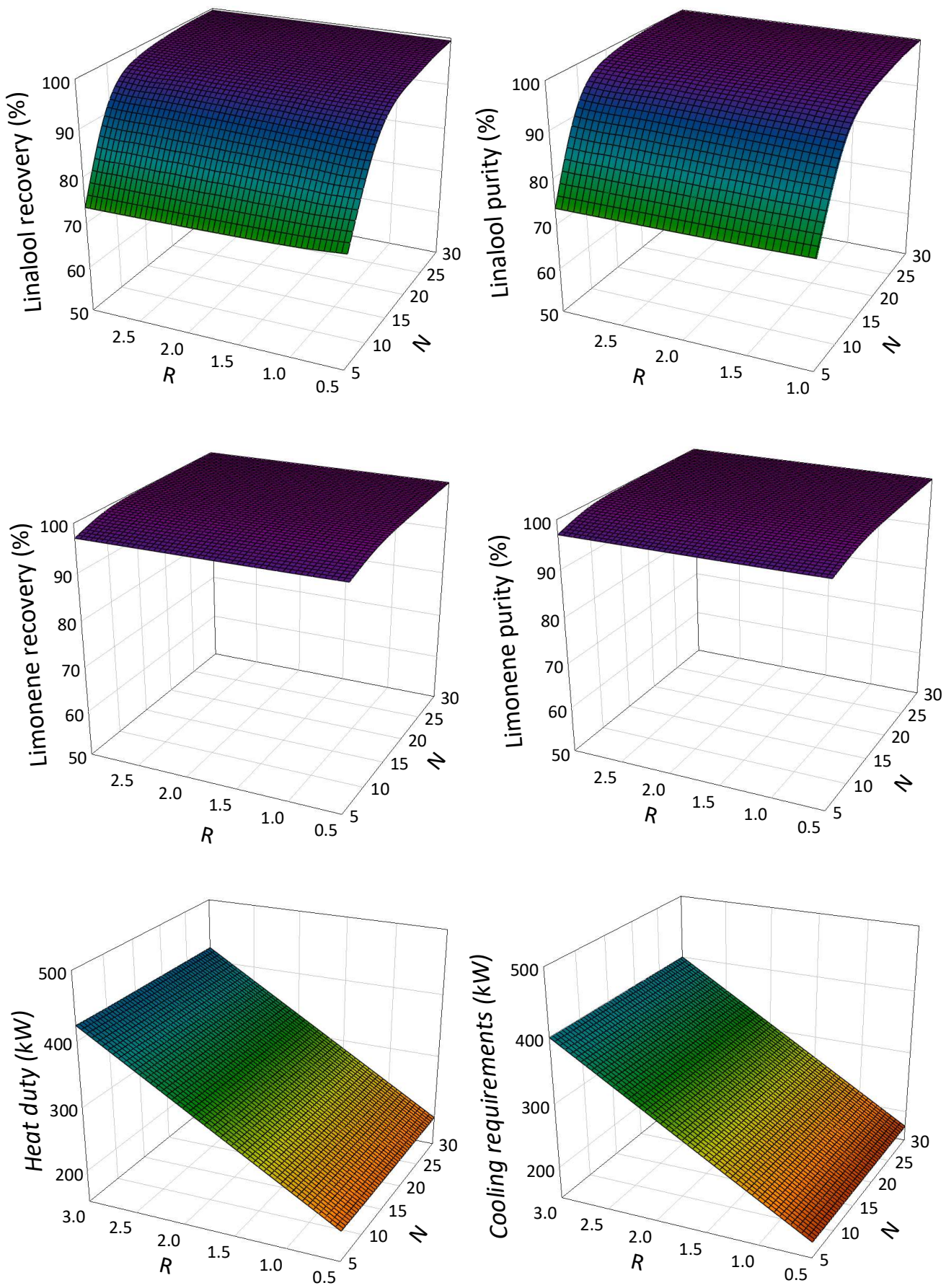

Figure S5. Linalool and limonene recovery and purity, heat duty and cooling requirements, as a function of the number of stages and reflux ratio in the extractive distillation column $(S / F=0.05)$, according to the schematic flowsheet shown in Figure 2 (main text). 


\section{References}

[S1] J.O. Valderrama. W.W. Sanga. J.A. Lazzús. Critical Properties. Normal Boiling Temperature. and Acentric Factor of Another 200 Ionic Liquids. Ind. Eng. Chem. Res. 47 (2008) 1318-1330. DOI: 10.1021/ie071055d.

[S2] J. Rudkin. Equation Predicts Vapor Pressures. Chem. Eng.: April 17 (1961) 202203

[S3] E. Zorebski. M. Musiał. K. Bałuszyńska. M.Zorębski. M. Dzida. Isobaric and Isochoric Heat Capacities as Well as Isentropic and Isothermal Compressibilities of Diand Trisubstituted Imidazolium-Based Ionic Liquids as a Function of Temperature. Ind. Eng. Chem. Res. 57 (2018) 5161-5172. DOI: 10.1021/acs.iecr.8b00506 